\title{
Sequences Generated by Polynomials over Integral Domains ${ }^{\dagger}$
}

\author{
Veasna KIM ${ }^{1}$, Vichian LAOHAKOSOL ${ }^{2}$ and Supawadee PRUGSAPITAK ${ }^{1, *}$ \\ ${ }^{1}$ Department of Mathematics and Statistics, Prince of Songkla University, Hat Yai 90110 Thailand \\ ${ }^{2}$ Department of Mathematics, Kasetsart University, Bangkok 10900 Thailand
}

(*Corresponding author's e-mail: supawadee.p@psu.ac.th)

Received: 30 November 2018, Revised: 19 February 2019, Accepted: 28 February 2019

\begin{abstract}
Let $D$ be an integral domain. For sequences $\bar{a}=\left(a_{1}, a_{2}, \ldots, a_{n}\right)$ and $I=\left(i_{1}, i_{2}, \ldots, i_{n}\right)$ in $D^{n}$ with distinct $i_{j}$, call $\bar{a}$ a $\left(D^{n}, I\right)$-polynomial sequence if there exists $f(x) \in D[x]$ such that $f\left(i_{j}\right)=a_{j}(j=$ $1, \ldots, n)$. Criteria for a sequence to be a $\left(D^{n}, I\right)$-polynomial sequence are established and explicit structures of $D^{n} / P_{n, I}$ where $P_{n, I}$ is the set of all $\left(D^{n}, I\right)$-polynomial sequences are determined.
\end{abstract}

Keywords: Polynomial sequences, sequence over integral domain, interpolation polynomials

Mathematics Subject Classification: 11B83, 11C08, 13G05

\section{Introduction}

For a fixed $n \in \mathbb{N}$, by a polynomial sequence (of length $n$ ), we mean a sequence $\bar{a}:=\left(a_{1}, a_{2}, \ldots ., a_{n}\right)$ in $\mathbb{Z}^{n}$ for which there exists $f(x) \in \mathbb{Z}[x]$ such that $f(i)=a_{i}$ for all $i=1,2, \ldots, n$; we refer to $f(x)$ as a polynomial which generates the sequence $\bar{a}$. Denote by $P_{n}$ the set of all polynomial sequences. Cornelius, Jr. and Schultz in [1] characterized $P_{n}$ using Lagrange and (implicitly) Newton interpolation polynomials and determined the structure of $\mathbb{Z}^{n} / P_{n}$.

The main objectives of this work are first to extend the characterization of Cornelius-Schultz from $\mathbb{Z}$ to an integral domain $D$ and second, to determine their corresponding structure.

Throughout, let $I=\left(i_{1}, i_{2}, \ldots, i_{n}\right) \in D^{n}$ with distinct $i_{j}$ and let

$P_{n, I}=\left\{\bar{a}=\left(a_{1}, \ldots, a_{n}\right) \in D^{n} \mid\right.$ there exists $f(x) \in D[x]$ such that $f\left(i_{j}\right)=a_{j}$ for all $\left.1 \leq j \leq n\right\}$

be the set of all $\left(D^{n}, I\right)$-polynomial sequences. It is easy to see that the set $P_{n, I}$ is a group under addition and if $\bar{a} \in P_{n, I}$ then $c \bar{a} \in P_{n, I}$ for any $c \in D$.

\section{Characterization}

For a fixed sequence $I$ as above and a sequence $\bar{a}:=\left(a_{1}, \ldots, a_{n}\right) \in D^{n}$, the Lagrange interpolation polynomial, [2, page 33], which interpolates the points $\left(i_{j}, a_{j}\right)(1 \leq j \leq n)$, is defined by

$L_{a, I}(x):=\sum_{j=1}^{n} a_{j} \prod_{m=1, m \neq j}^{n} \frac{x-i_{m}}{i_{j}-i_{m}} \in D_{Q}[x] \quad\left(D_{Q}\right.$ the quotient field of $\left.D\right)$

and satisfies

$L_{a, I}\left(i_{j}\right)=a_{j}(1 \leq j \leq n)$.

†resented at the International Conference in Number Theory and Applications 2018: December $13^{\text {th }}-15^{\text {th }}, 2018$ 
Theorem 1. Let $I=\left(i_{1}, i_{2}, \ldots, i_{n}\right) \in D^{n}$ with distinct $i_{j}$. Then $\bar{a}=\left(a_{1}, \ldots, a_{n}\right) \in D^{n}$ is a $\left(D^{n}, I\right)$ polynomial sequence if and only if $L_{a, I}(x) \in D[x]_{n}$, the set of all polynomials in $D[x]$ of degree $<n$. Furthermore, $L_{a, I}(x)$ is the unique polynomial of degree $<n$ in $D_{Q}[x]$ that generates $\bar{a}$.

Proof. If $\bar{a} \in P_{n, I}$, then there is $f(x) \in D[x]$ such that $f\left(i_{j}\right)=a_{j}(1 \leq j \leq n)$. We next let a polynomial $p(x):=\left(x-i_{1}\right) \cdots\left(x-i_{n}\right) \in D[x], \operatorname{deg} p(x)=n$. Since $p(x)$ is monic, by the division algorithm, $f(x)=q(x) p(x)+r(x)$, where $q, r \in D[x]$ with $\operatorname{deg} r<n$. Evaluating at the points $i_{j}(1 \leq j \leq n)$, we see that $r(x)$ generates the sequence $\bar{a}$ which shows that both $r(x)$ and $L_{a, I}(x)$ are polynomials in $D_{Q}[x]$ of degree $<n$ which agree at $n$ distinct points and so both must be identical. The remaining assertions are trivial.

Taking $I=(1,2, \ldots, n)$ in Theorem 1 , we recover [1, Theorem 2.1].

Given a set of $n$ points $\left(i_{k}, a_{k}\right)(k=1, \ldots, n)$, with distinct $i_{k}$ and $a_{k}$ being in $D$, the Newton interpolation polynomial corresponding to the points $\left(i_{k}, a_{k}\right)(k=1, \ldots, n)$ is defined as

$N_{a, I}(x)=b_{0, I}+b_{1, I}\left(x-i_{1}\right)+b_{2, I}\left(x-i_{1}\right)\left(x-i_{2}\right)+\cdots+b_{n-1, I}\left(x-i_{1}\right)\left(x-i_{2}\right) \cdots\left(x-i_{n-1}\right)$,

where $b_{k, I}=\sum_{j=0}^{k} \frac{a_{j+1}}{\prod_{m=1, m \neq j+1}^{k+1}\left(i_{j+1}-i_{m}\right)} \quad(0 \leq k \leq n-1)$. Note that the Newton interpolation polynomial can be obtained by solving the system of equations

$N_{a, I}\left(i_{k}\right)=a_{k} \quad(1 \leq k \leq n)$

which can be done inductively. The elements

$1, p_{i_{1}}:=\left(x-i_{1}\right), p_{i_{2}}:=\left(x-i_{1}\right)\left(x-i_{2}\right), \ldots, p_{i_{n-1}}:=\left(x-i_{1}\right)\left(x-i_{2}\right) \cdots\left(x-i_{n}\right)$

are referred to as the corresponding Newton basis polynomials [2, page 39-40].

Theorem 2. With the above notations, we have

$N_{a, I}(x)=L_{a, I}(x)$

Proof. By Theorem 1, $L_{a, I}(x)$ is the unique polynomial with coefficients in $D_{Q}$ of degree less than $n$ generating $\bar{a}$. Since $N_{a, I}\left(i_{j}\right)=a_{j}=L_{a, I}\left(i_{j}\right)$ for $1 \leq j \leq n$ and $\operatorname{deg} N_{a, I}<n$, they are identical.

Corollary 3. Let $\bar{a} \in D^{n}$. Then $\bar{a} \in P_{n, I}$ if and only if

$b_{k, I}=\sum_{j=0}^{k} \frac{a_{j+1}}{\prod_{m=1, m \neq j+1}^{k+1}\left(i_{j+1}-i_{m}\right)} \quad(k=0,1, \ldots, n-1)$

is an element in $D$.

Proof. The result follows immediately from Theorems 1 and 2.

Taking $I=(1,2,3, \ldots, n)$ in Theorems 1 and 2 , we get the following corollary. 
Corollary 4. Let $\bar{a} \in \mathbb{Z}^{n}$.

A) ([1, Lemma 2.2]) If $N_{a}(x)=b_{0} p_{0}(x)+b_{1} p_{1}(x)+\cdots+b_{n-1} p_{n-1}(x), b_{k}=\sum_{j=0}^{k} \frac{(-1)^{k+j}}{j !(k-j) !} a_{j+1}$ $(k=0, \ldots, n-1)$, then

$N_{a}(x)=L_{a}(x)$.

B) ([1, Corollary 2.4]) A sequence $\bar{a}$ is a polynomial sequence if and only if each number

$b_{k}=\sum_{j=0}^{k} \frac{(-1)^{k+j} a_{j+1}}{j !(k-j) !} \quad(k=0,1, \ldots, n-1)$

is an integer. result.

It is of interest to investigate the above results for small values of $n$. Thus we obtain the following

Lemma 5. With the above notations, the following statements hold:

A) For any $I=\left(i_{1}\right) \in \mathbb{Z}$, we have $P_{1, I}=\mathbb{Z}$.

B) For any $\bar{a}=\left(a_{1}, a_{2}\right), I=\left(i_{1}, i_{2}\right) \in \mathbb{Z}^{2}$ where $i_{1}<i_{2}$, we have

$$
\bar{a} \in P_{2, I} \quad \text { if and only if } a_{1} \equiv a_{2} \quad \bmod \left(i_{1}-i_{2}\right) .
$$

In fact, if $I=(1,2)$, then $P_{2}=\mathbb{Z}^{2}$.

C) For any $\bar{a}=\left(a_{1}, a_{2}, a_{3}\right), I=\left(i_{1}, i_{2}, i_{3}\right) \in \mathbb{Z}^{3}$ where $i_{1}<i_{2}<i_{3}$, we have

$$
\bar{a} \in P_{3, I} \quad \text { if and only if } \quad \frac{\left(a_{3}-a_{2}\right)+m\left(i_{2}-i_{3}\right)}{\left(i_{1}-i_{3}\right)\left(i_{2}-i_{3}\right)} \quad \text { and } \quad m=\frac{a_{1}-a_{2}}{i_{1}-i_{2}} \quad \text { are integers. }
$$

In fact, if $I=(1,2,3)$, then $P_{3}=\left\{\left(a_{1}, a_{2}, a_{3}\right) \in \mathbb{Z}^{3} \mid a_{1} \equiv a_{3} \bmod 2\right\}$.

Proof. We prove the above results as follows:

A) For any $a \in \mathbb{Z}$ there exists $f(x)=a$ such that $f\left(i_{1}\right)=a$. Thus $P_{1, I}=\mathbb{Z}$.

B) Let $\bar{a}=\left(a_{1}, a_{2}\right) \in \mathbb{Z}^{2}$. By Corollary $3, \bar{a} \in P_{2, I}$ if and only if $b_{0, I}=a_{1}$ and $b_{1, I}=\frac{a_{1}}{i_{1}-i_{2}}+\frac{a_{2}}{i_{2}-i_{1}}=$ $\frac{a_{1}-a_{2}}{i_{1}-i_{2}}$ are integers. Hence, $\bar{a} \in P_{2, I}$ if and only if $a_{1} \equiv a_{2} \bmod \left(i_{1}-i_{2}\right)$. If $I=(1,2)$, then $i_{1}-i_{2}=1$, and so $P_{2}=\mathbb{Z}^{2}$.

C) Let $\bar{a}=\left(a_{1}, a_{2}, a_{3}\right) \in \mathbb{Z}^{3}$. Then

$b_{0, I}=a_{1}$,

$b_{1, I}=\frac{a_{1}}{i_{1}-i_{2}}+\frac{a_{2}}{i_{2}-i_{1}}=\frac{a_{1}-a_{2}}{i_{1}-i_{2}}$,

$b_{2, I}=\frac{a_{1}}{\left(i_{1}-i_{2}\right)\left(i_{1}-i_{3}\right)}+\frac{a_{2}}{\left(i_{2}-i_{1}\right)\left(i_{2}-i_{3}\right)}+\frac{a_{3}}{\left(i_{3}-i_{1}\right)\left(i_{3}-i_{2}\right)}=\frac{\left(a_{3}-a_{2}\right)+m\left(i_{2}-i_{3}\right)}{\left(i_{1}-i_{3}\right)\left(i_{2}-i_{3}\right)}$,

where $m=\frac{a_{1}-a_{2}}{i_{1}-i_{2}}$.

By Corollary $3, \bar{a} \in P_{3, I}$ if and only if $m=\frac{a_{1}-a_{2}}{i_{1}-i_{2}} \in \mathbb{Z}$ and $\frac{\left(a_{3}-a_{2}\right)+m\left(i_{2}-i_{3}\right)}{\left(i_{1}-i_{3}\right)\left(i_{2}-i_{3}\right)}$ are integers . If $I=(1,2,3)$, then $m=\frac{a_{1}-a_{2}}{1-2}=a_{2}-a_{1}$ is an integer. Hence,

$\frac{\left(a_{3}-a_{2}\right)+m(2-3)}{(1-3)(2-3)}=\frac{\left(a_{3}-a_{2}\right)+\left(a_{2}-a_{1}\right)(-1)}{2}=\frac{a_{3}-a_{1}}{2}-a_{2}$ 
is an integer if and only if $2 \mid a_{3}-a_{1}$. Thus $\bar{a} \in \mathbb{Z}^{3}$ is a polynomial sequence of length 3 if and only if $a_{1}$ and $a_{3}$ are of the same parity.

The next result shows how to turn a sequence into a $\left(D^{n}, I\right)$-polynomial sequence.

Theorem 6. Let $I=\left(i_{1}, i_{2}, \ldots, i_{n}\right) \in D^{n}$ with distinct $i_{j}$, let $\bar{a}=\left(a_{1}, a_{2}, \ldots, a_{n}\right) \in D^{n}$ and let

$M=\prod_{j=0}^{n-1} M_{j}, \quad$ where $\quad M_{j}=\prod_{m=1, m \neq j+1}^{n}\left(i_{j+1}-i_{m}\right)(j=0,1,2, \ldots, n-1)$.

Then $M \bar{a}=\left(M a_{1}, M a_{2}, \ldots, M a_{n}\right) \in P_{n, I}$.

Moreover, if $D$ is a unique factorization domain, then $M^{\prime} \bar{a}=\left(M^{\prime} a_{1}, M^{\prime} a_{2}, \ldots, M^{\prime} a_{n}\right) \in P_{n, I}$ where $M^{\prime}=\operatorname{lcm}\left\{M_{j}\right\}_{j=0}^{n-1}$ and $M^{\prime}$ is the minimal element in $D$ for which this is true for every sequence of length $n$. The element $M^{\prime}$ is the minimal in the sense that if $L \bar{a} \in P_{n, I}$ for all $n$ then $M^{\prime} \mid L$.

Proof. Using the above notation, since

$b_{k, I}=\sum_{j=0}^{k} \frac{a_{j+1}}{\prod_{m \neq j+1, m=1}^{k+1}\left(i_{j+1}-i_{m}\right)}=\sum_{j=0}^{k} \frac{a_{j+1}}{M_{j} / \prod_{m=k+2, m \neq j+1}^{n}\left(i_{j+1}-i_{m}\right)}(0 \leq k \leq n-1)$,

we see that $M b_{k, I} \in \mathbb{Z}$ and so $M \bar{a}$ is a $\left(D^{n}, I\right)$-polynomial sequence. in $D$.

If $D$ is a unique factorization domain, then letting $M^{\prime}=\operatorname{lcm}\left\{M_{j}\right\}_{j=0}^{n-1}$, it is easy to see that $M^{\prime} b_{k, I}$ is

To see that $M^{\prime}$ is the minimal element with the stated property, consider the following sequences in Table 1.

Table 1 Sequences and its corresponding coefficients in the Newton interpolation polynomial

\begin{tabular}{cccccc}
\hline Sequence $\bar{a}$ & $b_{0, I}$ & $b_{1, I}$ & $b_{2, I}$ & $\ldots$ & $b_{n-1, I}$ \\
\hline $\bar{a}_{1}=(1,0,0, \ldots, 0)$ & 1 & $\frac{1}{i_{1}-i_{2}}$ & $\frac{1}{\left(i_{1}-i_{2}\right)\left(i_{1}-i_{3}\right)}$ & $\cdots$ & $\frac{1}{\left(i_{1}-i_{2}\right)\left(i_{1}-i_{3}\right) \cdots\left(i_{1}-i_{n}\right)}$ \\
$\bar{a}_{2}=(0,1,0, \ldots, 0)$ & 0 & $\frac{1}{i_{2}-i_{1}}$ & $\frac{1}{\left(i_{2}-i_{1}\right)\left(i_{2}-i_{3}\right)}$ & $\cdots$ & $\frac{1}{\left(i_{2}-i_{1}\right)\left(i_{2}-i_{3}\right) \cdots\left(i_{2}-i_{n}\right)}$ \\
$\bar{a}_{3}=(0,0,1, \ldots, 0)$ & 0 & 0 & $\frac{1}{\left(i_{3}-i_{1}\right)\left(i_{3}-i_{2}\right)}$ & $\cdots$ & $\frac{1}{\left(i_{3}-i_{1}\right)\left(i_{3}-i_{2}\right)\left(i_{3}-i_{4}\right) \cdots\left(i_{3}-i_{n}\right)}$ \\
$\vdots$ & $\vdots$ & $\vdots$ & $\vdots$ & $\vdots$ & $\vdots$ \\
$\bar{a}_{n}=(0,0,0, \ldots, 1)$ & 0 & 0 & 0 & $\cdots$ & $\frac{1}{\left(i_{n}-i_{1}\right)\left(i_{n}-i_{2}\right) \cdots\left(i_{n}-i_{n-1}\right)}$ \\
\hline
\end{tabular}

For each $\bar{a}_{i}(1 \leq i \leq n)$, we see that $M_{i-1} \bar{a}_{i} \in P_{n, I}$ and for any element $L \in D$ such that $L \bar{a}_{i} \in P_{n, I}$, we have $M_{i-1} \mid L(1 \leq i \leq n)$. Therefore, by the definition of $M^{\prime}$, we have $M^{\prime} \mid L$, showing that $M^{\prime}$ is the minimal element such that $M^{\prime} \bar{a} \in P_{n, I}$.

Before proceeding, let us work out two examples.

Example 1. a) Let $D=\mathbb{Z}, \bar{a}=(2,8,12)$ and $I=(5,6,8)$. We see that

$N_{a, I}(x)=-\frac{4}{3} x^{2}+\frac{62}{3} x-68 \notin \mathbb{Z}[x]$. 
So $\bar{a} \notin P_{3, I}$ over $\mathbb{Z}$. Since $M_{0}=3, M_{1}=2$ and $M_{2}=6, M^{\prime}=6$. We deduce that $M^{\prime} \bar{a}=(12,48,72)$ is a polynomial sequence generated by $-8 x^{2}+24 x-408$ with respect to $I=(5,6,8)$ in $\mathbb{Z}$.

b) Let $\bar{c}=(4-i, 5,6+2 i) \in \mathbb{Z}[i]^{3}$ and $I=(i, 3 i, 2+i) \in \mathbb{Z}[i]^{3}$. We see that

$N_{c, I}(x)=\frac{-9+13 i}{8} x^{2}+(7+4 i) x+\frac{55-51 i}{8} \notin \mathbb{Z}[i][x]$.

So $\bar{c} \notin P_{3, I}$ over $\mathbb{Z}[i]$. Since $M_{0}=-4 i, M_{1}=-4(1+i)$ and $M_{2}=4(1-i), M^{\prime}=8$, we get that $M^{\prime} \bar{c}=(32-8 i, 40,48+16 i)$ is a polynomial sequence generated by $(-3+5 i) x^{2}+(24+8 i) x+(37-27 i)$ with respect to $I=(i, 3 i, 2+i)$ in $\mathbb{Z}[i]$.

If $D=\mathbb{Z}$ and $I=(1,2, \ldots, n)$, then we have the following result which is [1, Theorem 2.5].

Corollary 7. If $a=\left(a_{1}, a_{2}, \ldots, a_{n}\right) \in \mathbb{Z}^{n}$, then

$(n-1) ! a=\left((n-1) ! a_{1},(n-1) ! a_{2}, \ldots,(n-1) ! a_{n}\right) \in P_{n, I}$.

Moreover, $(n-1) !$ is the least positive integer for which this is true for every sequence of length $n$.

Proof. Take $I=(1,2,3, \ldots, n)$. Using the same notation as in Theorem 6, we compute

$M_{j}=\prod_{m=1, m \neq j+1}^{n}(j+1-m)=(-1)^{n-j-1}(j) !(n-j-1) !(0 \leq j \leq n-1)$.

Since $(n-1) !=(j) !(n-j-1) !\left(\begin{array}{c}n-1 \\ j\end{array}\right)(0 \leq j \leq n-1)$, the integer $M_{j}$ is a divisor of $(n-1) !$ for all $0 \leq j \leq n-1$ and $M_{n-1}=(n-1)$ !. Hence, $M=\operatorname{lcm}\left(M_{1}, M_{2}, \ldots, M_{n}\right)=(n-1)$ !.

\section{Structure}

In this section, we show that $P_{n, I}$ is a rank $n$ subgroup of the free abelian group $D^{n}$. We first show that for any $I \in D^{n}$, we have $P_{n, I} \cong D[x]_{n}$ as a group where $D[x]_{n}$ is the set of polynomial in $D[x]$ of degree less than $n$.

Theorem 8. The group $P_{n, I}$ is isomorphic to $D[x]_{n}$.

Proof. Define $v: D[x] \longrightarrow D^{n}$ by $v(f(x))=\left(f\left(i_{1}\right), f\left(i_{2}\right), \ldots, f\left(i_{n}\right)\right)$. Let $f_{1}, f_{2} \in D[x]_{n}$. Then

$$
\begin{aligned}
v\left(\left(f_{1}+f_{2}\right)(x)\right) & =\left(\left(f_{1}+f_{2}\right)\left(i_{1}\right),\left(f_{1}+f_{2}\right)\left(i_{2}\right), \ldots,\left(f_{1}+f_{2}\right)\left(i_{n}\right)\right) \\
& =\left(f_{1}\left(i_{1}\right)+f_{2}\left(i_{1}\right), f_{1}\left(i_{2}\right)+f_{2}\left(i_{2}\right), \ldots, f_{1}\left(i_{n}\right)+f_{2}\left(i_{n}\right)\right)=v\left(f_{1}(x)\right)+v\left(f_{2}(x)\right) .
\end{aligned}
$$

Thus $v$ is an additive homomorphism. We next show that $v$ restricted to $D[x]_{n}$ is an isomorphism from $D[x]_{n}$ to $P_{n, I}$. Let $\bar{a}=\left(a_{1}, a_{2}, \ldots, a_{n}\right) \in P_{n, I}$. Then there exists $f(x) \in D[x]$ such that $f(x)$ generates $\bar{a}$. Again as in Theorem 1, $f(x)=q(x) p(x)+r(x)$ where $p(x)=\left(x-i_{1}\right) \cdots\left(x-i_{n}\right), q, r \in D[x]$ with $r=0$ or $\operatorname{deg} r<n$. Evaluating at the points $i_{j}(1 \leq j \leq n)$, we see that $r(x)$ generates the sequence $\bar{a}$. So $v$ is onto.

Let $f, g \in D[x]_{n}$. Suppose $v(f(x))=v(g(x))$. Then $f\left(i_{k}\right)=g\left(i_{k}\right)$ for all $1 \leq k \leq n$. Since both $\operatorname{deg}(f)$ and $\operatorname{deg}(g)$ are $<n$ and the polynomials $f, g$ agree at $n$ distinct points, they are identical, i.e., $v$ is one-to-one. Therefore $v$ is an isomorphism from $D[x]_{n}$ onto $P_{n, I}$. 
We next consider the structure of $\mathbb{Z}^{n} / P_{n, I}$. For $I=(1,2, \ldots, n) \in \mathbb{Z}^{n}$, it was shown in $[1$, Theorem 3.2] that

$\mathbb{Z}^{n} / P_{n} \cong \mathbb{Z} / 2 ! \mathbb{Z} \oplus \mathbb{Z} / 3 ! \mathbb{Z} \oplus \cdots \oplus \mathbb{Z} /(n-1) ! \mathbb{Z}$.

We use the technique similar to that in [1] to generalize the above result to $D^{n} / P_{n, I}$.

Theorem 9. For $n \geq 2$, let $I=\left(i_{1}, i_{2}, \ldots, i_{n}\right) \in D^{n}$. If

$\prod_{m=1}^{k-1}\left(i_{j}-i_{m}\right) / \prod_{m=1}^{k-1}\left(i_{k}-i_{m}\right) \in D \quad(1<k<j \leq n)$,

then

$D^{n} / P_{n, I} \cong D /\left(i_{2}-i_{1}\right) D \oplus D /\left(i_{3}-i_{1}\right)\left(i_{3}-i_{2}\right) D \oplus \cdots \oplus D /\left(i_{n}-i_{1}\right)\left(i_{n}-i_{2}\right) \cdots\left(i_{n}-i_{n-1}\right) D$.

Proof. For $j, k \in\{1,2, \ldots, n\}$, let

$a_{j k}= \begin{cases}\prod_{m=1}^{k-1}\left(i_{j}-i_{m}\right) / \prod_{m=1}^{k-1}\left(i_{k}-i_{m}\right) & \text { if } j \geq k>1 \\ 1 & \text { if } k=1 \\ 0 & \text { if } j<k,\end{cases}$

so that

$A_{n}=\left(a_{j k}\right)=\left[\begin{array}{cccccc}1 & 0 & 0 & 0 & \ldots & 0 \\ 1 & 1 & 0 & 0 & \ldots & 0 \\ 1 & \frac{i_{3}-i_{1}}{i_{2}-i_{1}} & 1 & 0 & \ldots & 0 \\ \vdots & \vdots & \vdots & \vdots & \ddots & \vdots \\ 1 & \frac{i_{n}-i_{1}}{i_{2}-i_{1}} & \frac{\left(i_{n}-i_{1}\right)\left(i_{n}-i_{2}\right)}{\left(i_{3}-i_{1}\right)\left(i_{3}-i_{2}\right)} & \frac{\left(i_{n}-i_{1}\right)\left(i_{n}-i_{2}\right)\left(i_{n}-i_{3}\right)}{\left(i_{4}-i_{1}\right)\left(i_{4}-i_{2}\right)\left(i_{4}-i_{3}\right)} & \ldots & 1\end{array}\right]$.

Let $e_{I}(j-1)$ be the $j^{\text {th }}$ column of $A_{n}(j=1,2, \ldots, n)$. Since $\operatorname{det} A_{n}=1$ and

$a_{j k}=\prod_{m=1}^{k-1}\left(i_{j}-i_{m}\right) / \prod_{m=1}^{k-1}\left(i_{k}-i_{m}\right) \in D \quad(1<k<j)$,

the matrix $A_{n}$ is a unimodular [3, Lemma 1.15]. In this case, we see that $\left\{e_{I}(j-1), j=1,2, \ldots, n\right\}$ forms a $D$-basis for $D^{n}$. Now let

$C_{n}=\left(c_{j k}\right)=\left[\begin{array}{ccccc}1 & 0 & 0 & \ldots & 0 \\ 1 & i_{2}-i_{1} & 0 & \ldots & 0 \\ 1 & i_{3}-i_{1} & \left(i_{3}-i_{1}\right)\left(i_{3}-i_{2}\right) & \ldots & 0 \\ \vdots & \vdots & \vdots & \ddots & \vdots \\ 1 & i_{n}-i_{1} & \left(i_{n}-i_{1}\right)\left(i_{n}-i_{2}\right) & \ldots & \left(i_{n}-i_{1}\right) \ldots\left(i_{n}-i_{n-1}\right)\end{array}\right]$,
$c_{j k}= \begin{cases}\left(i_{j}-i_{1}\right)\left(i_{j}-i_{2}\right) \cdots\left(i_{j}-i_{k-1}\right) & \text { if } 1<k \leq j \\ 1 & \text { if } k=1 \\ 0 & \text { if } j<k,\end{cases}$

and let $D_{n}$ be the diagonal matrix whose $j^{\text {th }}$ diagonal entries are

$d_{j, I}=\left(i_{j}-i_{1}\right)\left(i_{j}-i_{2}\right) \cdots\left(i_{j}-i_{j-1}\right) \quad(j=1,2, \ldots, n)$. 
It is easy to see that $C_{n}=A_{n} D_{n}$. Since $\left\{1, p_{i_{1}}(x), \ldots, p_{i_{n-1}}(x)\right\}$ forms a $D$-basis for $D[x]_{n}$, by Theorem 8 , the map $v: D[x]_{n} \longrightarrow P_{n, I}$ is an isomorphism. So the image

$\left\{v(1), v\left(p_{i_{1}}(x)\right), \ldots, v\left(p_{i_{n-1}}(x)\right)\right\}$

forms a $D$-basis for $P_{n, I}$. From

$v\left(p_{i_{0}}(x)\right)=\left[\begin{array}{c}1 \\ 1 \\ \vdots \\ 1\end{array}\right], v\left(p_{i_{1}}(x)\right)=\left[\begin{array}{c}0 \\ i_{2}-i_{1} \\ i_{3}-i_{1} \\ \vdots \\ i_{n}-i_{1}\end{array}\right], \ldots, v\left(p_{i_{n-1}}(x)\right)=\left[\begin{array}{c}0 \\ 0 \\ 0 \\ \vdots \\ \left(i_{n}-i_{1}\right)\left(i_{n}-i_{2}\right) \ldots\left(i_{n}-i_{n-1}\right)\end{array}\right]$,

we see that $v\left(p_{i_{j-1}}(x)\right)$ is the $j^{\text {th }}$ column of $C_{n}(j=1,2, \ldots, n)$. Since $C_{n}=A_{n} D_{n}$, we have

$\left(p_{i_{j-1}}(x)\right)=\left(i_{j}-i_{1}\right)\left(i_{j}-i_{2}\right) \cdots\left(i_{j}-i_{j-1}\right) e_{I}(j-1)=\prod_{m=1}^{j-1}\left(i_{j}-i_{m}\right) e_{I}(j-1)(j=1,2, \ldots, n)$.

Thus,

$$
\begin{aligned}
D^{n} / P_{n, I} & =\frac{\left\langle e_{I}(0)\right\rangle \oplus\left\langle e_{I}(1)\right\rangle \oplus\left\langle e_{I}(2)\right\rangle \oplus \cdots \oplus\left\langle e_{I}(n-1)\right\rangle}{\left\langle e_{I}(0)\right\rangle \oplus \prod_{m=1}^{1}\left(i_{2}-i_{m}\right)\left\langle e_{I}(1)\right\rangle \oplus \cdots \oplus \prod_{m=1}^{n-1}\left(i_{n}-i_{m}\right)\left\langle e_{I}(n-1)\right\rangle} \\
& =\frac{\left\langle e_{I}(0)\right\rangle}{\left\langle e_{I}(0)\right\rangle} \oplus \frac{\left\langle e_{I}(1)\right\rangle}{\prod_{m=1}^{1}\left(i_{2}-i_{m}\right)\left\langle e_{I}(1)\right\rangle} \oplus \cdots \oplus \frac{\left\langle e_{I}(n-1)\right\rangle}{\prod_{m=1}^{n-1}\left(i_{n}-i_{m}\right)\left\langle e_{I}(n-1)\right\rangle} \\
& \cong D /\left(i_{2}-i_{1}\right) D \oplus D / \prod_{m=1}^{n}\left(i_{3}-i_{m}\right) D \oplus \cdots \oplus D / \prod_{m=1}^{n-1}\left(i_{n}-i_{m}\right) D .
\end{aligned}
$$

By Theorem 9, for $1 \leq j \leq n$, if $a_{j k}=\prod_{m=1}^{k-1}\left(i_{j}-i_{m}\right) / \prod_{m=1}^{k-1}\left(i_{k}-i_{m}\right) \in D \quad(1<k \leq j)$, choosing $k=j-1$, we get

$a_{j, j-1}=\prod_{m=1}^{j-2}\left(i_{j}-i_{m}\right) / \prod_{m=1}^{j-2}\left(i_{j-1}-i_{m}\right) \in D \quad(j=0,1, \ldots, n-1)$.

Thus, $d_{j, I}=\prod_{m=1}^{j-1}\left(i_{j}-i_{m}\right)=a_{j, j-1} \cdot\left(i_{j}-i_{j-1}\right) \cdot d_{j-1, I}$, i.e., $d_{j-1, I}$ is the factor of $d_{j, I}(j=1,2, \ldots, n)$, yielding

Corollary 10. With the set up above, $D^{n} / P_{n, I}$ is a finite abelian group of the form

$D / d_{n-1} D \oplus \cdots \oplus D / d_{2} D \oplus D / d_{1} D$

where $d_{1}\left|d_{2}\right| \cdots \mid d_{n-1}$.

If we take $D=\mathbb{Z}$ and $I=(1,2, \ldots, n)$, we deduce the following result.

Corollary 11. [1, Corollary 3.3] If $I=(1,2, \ldots, n) \quad(n \geq 3)$, then $\mathbb{Z}^{n} / P_{n}$ is a finite abelian group with Smith normal form

$\mathbb{Z} /(n-1) ! \mathbb{Z} \oplus \cdots \oplus \mathbb{Z} / 3 ! \mathbb{Z} \oplus \mathbb{Z} / 2 ! \mathbb{Z}$

and Smith invariant $((n-1) !, \ldots, 3 !, 2 !)$. Moreover, $\left|\mathbb{Z}^{n} / P_{n}\right|=\prod_{i=1}^{n-1} i$ !. 
We pause to look at one simple example.

Example 2. Let $D=\mathbb{Z}[i]$ and $I=(2+i, 3+4 i, 2+11 i)$. Since

$a_{3,2}=\frac{i_{3}-i_{1}}{i_{2}-i_{1}}=\frac{(2+11 i)-(2+i)}{(3+4 i)-(2+i)}=3+i \in \mathbb{Z}[i]$,

all the elements $a_{j k}$ of the matrix $A_{3}$ are in $\mathbb{Z}[i]$. By Theorem 9 we get

$\mathbb{Z}[i]^{3} / P_{3, I} \cong \frac{\mathbb{Z}[i]}{(1+3 i) \mathbb{Z}[i]} \oplus \frac{\mathbb{Z}[i]}{(10 i)(-1+7 i) \mathbb{Z}[i]}=\frac{\mathbb{Z}[i]}{(1+3 i) \mathbb{Z}[i]} \oplus \frac{\mathbb{Z}[i]}{(-70-10 i) \mathbb{Z}[i]}$.

The quotient condition in Theorem 9 simplifies for some particular sets $I$ as witnessed in the next corollary.

Corollary 12. The following statments hold:

A) Let $a, q$ be elements in $D$ and $n \geq 2$. If $i_{k}=a q^{k}(1 \leq k \leq n)$, then

$D^{n} / P_{n, I} \cong D / a q(q-1) D \oplus D / a^{2} q^{1+2}\left(q^{2}-1\right)(q-1) D \oplus \cdots \oplus D / a^{n-1} q^{1+2+3+\cdots+(n-1)} \prod_{i=1}^{n-1}\left(q^{i}-1\right) D$.

B) For $n \geq 2,1 \leq k \leq n-1$, if $i_{k+1}-i_{k}=$ for some $c \in D$, then

$D^{n} / P_{n, I} \cong D / c \cdot D \oplus D / 2 ! c^{2} D \oplus D / 3 ! c^{3} D \oplus \cdots \oplus D /(n-1) ! c^{n-1} D$.

Proof. A) Since $i_{k}=a q^{k}, i_{k+1}-i_{k}=a q^{k}(q-1)(1 \leq k \leq n-1)$, we have $i_{j}-i_{k}=a q^{j}-a q^{k}=$ $a q^{k}\left(q^{j-k}-1\right)(j>k)$. By the proof of Theorem 9, we get

$A_{n}=\left(a_{j k}\right), \quad a_{j k}= \begin{cases}\frac{\prod_{m=1}^{k-1}\left(i_{j}-i_{m}\right)}{\prod_{m=1}^{k-1}\left(i_{k}-i_{m}\right)}=\frac{\prod_{m=1}^{k-1}\left(q^{j-m}-1\right)}{\prod_{m=1}^{k-1}\left(q^{m}-1\right)} & \text { if } j \geq k>1 \\ 1 & \text { if } k=1 \\ 0 & \text { if } j<k .\end{cases}$

For $1 \leq k \leq j \leq n$, since $\prod_{m=1}^{k-1}\left(q^{j-m}-1\right) / \prod_{m=1}^{k-1}\left(q^{m}-1\right)$ is a $q$-binomial coefficient, it is in $D$ and by Theorem 9 we have

$D^{n} / P_{n, I} \cong D / a q(q-1) D \oplus D / a^{2} q^{3}\left(q^{2}-1\right)(q-1) D \oplus \cdots \oplus D / a^{n-1} q^{\frac{n(n-1)}{2}} \prod_{i=1}^{n-1}\left(q^{i}-1\right) D$.

B) Since $i_{k+1}-i_{k}=c(1 \leq k \leq n-1)$, we have

$i_{j}-i_{k}=\left(i_{j}-i_{j-1}\right)+\left(i_{j-1}-i_{j-2}\right)+\cdots+\left(i_{k+1}-i_{k}\right)=(j-k) c(j>k)$.

By the proof of Theorem 9, we get

$A_{n}=\left(a_{j k}\right), \quad a_{j k}= \begin{cases}\prod_{m=1}^{k-1}\left(i_{j}-i_{m}\right) / \prod_{m=1}^{k-1}\left(i_{k}-i_{m}\right)=\left(\begin{array}{c}j-1 \\ k-1\end{array}\right) & \text { if } j \geq k>1 \\ 1 & \text { if } k=1 \\ 0 & \text { if } j<k\end{cases}$ 
Thus, $a_{j k} \in D$ and by Theorem 9, it is easy to see that

$D^{n} / P_{n, I} \cong D / c D \oplus D / 2 ! c^{2} D \oplus \cdots \oplus D /(n-1) ! c^{n-1} D$.

Taking $D=\mathbb{Z}, I=\{1,2, \ldots, n\}$ and $c=1$ in Corollary 12 B), we recover [1, Theorem 3.2].

\section{Acknowledgements}

We are grateful to the referees for their valuable comments and suggestions to improve this article. The research is supported by Faculty of Science, Prince of Songkla University, Thailand.

\section{References}

[1] EF Cornelius Jr and P Schultz. Sequences generated by polynomials. Amer. Math. Monthly 2008; 115, 154-8.

[2] PJ Davis. Interpolation and Approximation. Dover, New York, 1975.

[3] I Stewart and D Tall. Algebraic Number Theory and Fermat's Last Theorem. CRC Press, 2001. 